\title{
Dynamics of artistic style: a computational analysis of the Maker's motoric qualities in a clay-relief practice
}

Nir Dick ${ }^{1}$, Ayala Prusak ${ }^{1} \&$ Amit Raphael Zoran ${ }^{1 凶}$

The artistic style has been extensively discussed within diverse perspectives, usually studying the physical qualities of existing artifacts as the resource for investigation. This paper proposes a novel analysis of the dynamics of artistic style, as represented by a set of motor features, techniques, and their temporal interplay. The researchers hypothesize that unique characteristics of individuals' styles are represented as transitions between motor activities, which would allow for computational analysis of style. As a case study, the researchers tracked a carving knife used in a clay-relief technique in two studies, one comprising (i) twelve sessions and five novice participants; and the other (ii) twenty-eight sessions with a single skilled artist. The analysis reveals that dynamic style is (i) unique and consistent in novices' creative processes and that (ii) different subcategories of making can be observed in an experienced participant related to the subject of the work. These offer the possibility of quantitatively studying the making process irrespective of the esthetic qualities of the finished artifact, which allows for diverse computational applications.

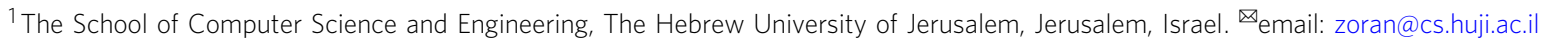




\section{Introduction}

ersonal qualities of artistic style leave unique marks on art products contributing to the extraordinary qualities of the artifacts by attributes such as textures and patterns (Davis, 1990); these have been the topic of studies in diverse academic disciplines (Summers, 2009; Schapiro, 1953). However, due to technological restraints, prior studies on personal artistic style neglect quantitative analyses of the making process as a function of time-variant motor activities. Moreover, although today computers allow for the successful synthesis of artists' styles, these studies tend to focus on statistically learning a portfolio of premade products rather than the making process itself.

Nevertheless, digital technologies enable us to detect, monitor, and classify dynamic characteristics of artists' ways of working: the real-time qualities and mathematical characteristics of the techniques they use, physical features identifying these techniques, and the statistical relationship between them.

In order to formulate a definition of dynamic style, we focus on personal style and motor skill, presenting a quantitative method to identify artistic style as a transition between motor states, and studying the motor performance of an artist working with a manual tool. The artist's tool-path is computationally examined to identify clusters of motor features, and the relationships between these clusters analyzed with respect to other artists or other projects by the same artist. Our method can thus be used to deepen our understanding of style and motor skill as the language of making (Davis, 1990: see section "Background on personal style"), supporting the future study of the dynamics of style, as well as the development of computational creative applications.

This paper presents a two-fold contribution: (1) a computational method to evaluate the dynamics of personal style; and (2) a proofof-concept of this method. In other words, we supply a technical approach to evaluate the unique and common motor features that construct one's way of making artifacts. These allow for the making process as a whole to be quantitatively observed as an interplay between various actions regardless of the esthetic qualities of the artifact. Hence, we analyze the work process itself instead of its final product-an analysis that is computationally simple, yet manually almost impossible, due to the amount and resolution of technical information generated during the working process.

As a case study, we used a magnetic motion tracking system (MMTS) to track (in $60 \mathrm{~Hz}$ ) the 6DOF location of a carving knife in a clay-relief technique, then processed and analyzed the recorded data using digital signal processing and machine learning tools. We conducted two studies. In the first, we tracked and analyzed the work of five novice participants, each performing three simple geometric tasks, demonstrating how one's style can be identified using our method. In the second, we tracked four complex works created by a professional sculptor executing two different work models, computationally showing that the artist's style depends greatly on the subject matter. We conclude that our method to study style as a transition between motor states has been proven to be valid, and raise questions for future research on the dynamics of style, with the aim of constructing a theoretical framework for further analysis of the topic.

\section{Motivation}

The creative artistic process is subjective, in many respects unconscious (Stevens, 2014), and depends on complex sociocultural and technical conditions (Summers, 2009; Schapiro, 1953). One approach to investigating the style of one's work is to consider it as a physical manifestation of the process (Wiessner, 1984).

Davis defines style as a description of a polythetic set of similar but varying attributes in a group of artifacts (1990, p. 19), and suggests that style can be seen as explaining the similarities between the attributes of artifacts. For Davis, style is the language of making, yet without the historical, social, and technological conditions for such communication, style cannot encode the contextual meaning it may represent.

Davis, who mostly discusses the style of pre-made artifacts, does not have access to the casual motor features that generate the attributes he mentions. To study style as a language, we need information regarding the temporal relationship between the attributes, techniques, and gestures produced by the maker. $A$ study of the dynamic qualities of style goes beyond a comparison between pre-made artifacts, to the study of the making process itself. A dynamic style approach allows for comparison between techniques as a function of the maker in the creative process and seeks correlations between working patterns within one's work and in between various makers. Hence, studying the dynamics of style allows us to generate comprehensive information about the "language of making," and how it changes and evolves over time.

Moreover, our motivation goes beyond a potential contribution to the theory of style. Today, the use of digital agents for cocreative purposes with humans is ubiquitous, but usually leads to the loss of some crucial aspects of the creative process (Zoran, 2016; Zoran et al., 2014a). Incorporating external technological agents adapted to the specific creative user's process (in the form of robotic apprentices assisting a master craftsperson, for instance), can help artists harness technology without losing vital aspects of the creative experience. To enable such technology, there is a need for a computational model of the artist's motor style of work (Zoran, 2016). We believe a dynamic approach to such style analysis lays the groundwork for developing collaborative technology that would create new avenues for creative experience and its products.

Today, with recent advances in machine learning (ML) and data analysis, people are accustomed to living in a personalized digital environment: watching television, shopping, listening to music (Linden et al., 2003)-all become highly personalized experiences. Tools for creative practice can offer the same sense of a personalized experience, provided the proper data and computational framework. Furthermore, the predictive abilities ML provides (Ghahramani, 2015) can be harnessed to infer an artist's future progress, given a temporal dynamic record of their style. As such, dynamic style research may enable technology to predict and synthesize artists' future styles before they have reached the skill level, or achieved the personal progress, to produce it themselves. This could redefine the meaning of practice and mastership while furthering the discussion of authenticity and artistic intent and ownership (Benjamin, 2018; Barthes, 1977).

Hybrid interaction and smart tools. The personal creative process can be viewed as a continuous feedback loop between mind and matter (Mace and Ward, 2002). Currently, most digital agents work exclusively at one end of the loop: fabrication and 3D-printing technologies replace direct contact between creator and material and focus on the final product. On the other hand, many computer-aided design platforms allow for planning the final product in a way that is separate from the manufacturing process, eliminating all material sensory feedback and replacing it with a digital interface. Digital technology is augmented to create tools with "smart" handles, which in some respects bridge the gap between digital representation and tangible interaction. Such synergy enables users to express and develop their personal style while being assisted by the digital agent (Zoran et al., 2014a).

By using digital agents, users outsource some of their creativity to an external agent. While artificial intelligence agents can style 
images as if they were painted by great artists (Jay et al., 2017), and even display a form of imagination (Mordvintsev et al., 2015), current stylization technology takes over creative control, filtering given inputs without collaborating with the human user (Zoran, 2016). A hybrid approach to interaction would leave space for the users to evolve and express their own style. However, such an approach would require accessing processes that are yet to be formalized in a computational way-the hidden control and feedback loops that construct the creative process.

Human-robot interaction. In recent years, the research field of human-robot interaction (HRI) has experienced significant growth. The computational frameworks and algorithms that enable communication between humans and robots keep evolving and setting new goals. These goals are developed and examined in the field of traditional robotics, but also through the prism of human social behavior and interaction (Thomaz et al., 2016). Incorporating robotics into the creative process, given its intimate nature, is essentially a delicate form of human-robot communication.

Humans and robots are working side-by-side, performing shared tasks, in many domains. Systems that engage in humanrobot collaboration call for the development of frameworks that will enable productive teamwork between people and machines. In such frameworks, the dynamic, non-deterministic human holds the main part of the robots' environment. In order to compute actions within such an environment, a psycho-cognitive approach is needed (Thomaz et al., 2016; Nikolaidis and Shah, 2012; Görür et al., 2017). Different computational frameworks are suggested to create such an operative dialog between people and robots, many of which suggest models for human intention, recognition, and prediction (Nikolaidis and Shah, 2012; Görür et al., 2017). When conceptualizing and building robots to take part in an artist's creative process, the challenges of human-robot collaboration become less tangible and more complex, as the creative process includes factors that the artists themselves may not be aware of (Stevens, 2014).

Having a "shared" mental model of a task promotes effective teamwork among humans and is thought to do the same in humanrobot collaboration (Nikolaidis and Shah, 2012). If the task is a creative one, the human artist's mental model of it is inferred from their individual creative process (Mace and Ward, 2002). In order to help machine companions learn and predict mental model features, it has been suggested that they be expressed as a sequence of actions over states (Nikolaidis and Shah, 2012). Metrics that allow for visualizing an artistic style manifest in a finite set of discrete techniques have the potential to set the groundwork for collaborative work with robots: dynamic style research supplies the computational tools required to teach robots to infer the model of an artistic task, and so collaborate in its making.

Our specific interest is in incorporating robotics into the domain of craft and creativity. This social domain is highly autonomous and liberal; as such, it requires a less deterministic and more flexible style of machine learning (de Miranda et al., 2016). Robots meant to work within a creative environment would need to learn how to improvise. For example, Shimon is an interactive robotic marimba player (Hoffman and Weinberg, 2010): it was specially designed to improvise jazz music, reacting to a human piano player in a joint performance. This robotic was modeled according to gestures rather than sounds, meaning that the robot learns from human dynamic actions, not from their static outcome. This embodied approach coincides with dynamic style analysis based on motor documentation of the artist's actions. Another robot working in the creative domain is "YOLO," a toy-like robot designed to boost creativity in children (Alves-Oliveira et al., 2017). YOLO is a social robot designed to inspire creative thinking in terms of innovation and problem solving, which is different from the more intimate, creative performance-oriented approach offered by dynamic style analysis and synthesis.

While many approaches to HRI apply a social framework to the relationship between humans and robots, a style-aware approach offers a more personal form of interaction in which the robot is less an external collaborative entity than an extension of the self. In addition, the use of $\mathrm{AI}$ and robotics suggests some autonomy, meaning that a style-aware robot might perform an independent reflection of the artist, which raises new questions about creativity and technology.

The question concerning style is complex and cannot be bounded within a controlled motoric investigation. However, focusing on the time-variant relationship between motor activities in a controlled environment, our investigation holds the potential to generate utilitarian insights regarding the synthesis of robotic application that imitates the motor technique of one's skills, allowing for tight and intimate human-robot interaction in complex creative tasks. While a complete discussion of the definitions and views of style is beyond our scope, a short overview is presented here to frame our work within this greater context.

\section{Background on personal style}

One can consider a range of complementary perspectives when studying style (Davis, 1990; Schapiro, 1953). Acts of artistic creation generate complex behavioral (Cobbledick, 1996), cognitive (Kozbelt, 2001; Winner and Casey, 1992), and motor (Glazek, 2012; Zoran et al., 2014b) interactions. Artists' signatures have been extracted from art products and studied (Gombrich, 1995; Rigau et al., 2008), while other researchers consider the interaction between style and culture (Clark, 2015). Summers argues that style, representing the autographic qualities of the artist, holds only a partial contribution to the product. For Summers, materiality, technology, and their historical and cultural context may have an even greater influence on the creative practice (2009).

Today, new technologies enable us to investigate the connections between such aspects of artists' work (Zoran et al., 2014b; Mordvintsev et al., 2015; Thomaz et al., 2016), and between artistic style and skill (Zoran et al., 2014b; Gandon et al., 2013), including computational analysis of the evolution of style over time in a controlled environment (Haroch et al., 2019). For our investigation, we adopt a view of style as a manner of doing something (Solso, 2001). We do not aim to contribute to the debate regarding the meaning of the term style but rely on a given view to better communicate our intention regarding the process and language of making. We are interested in motoric mechanisms and gestural causality that can contribute to a theoretical (quantitative) framework around personal artistic style.

Style in anthropology. Boas defined style as the consistent and formal elements of esthetics that appear in culture (Stevenson, 2010), focusing on empiricism and a historical point of view (ElOr, 2014; Boas, 1995), rather than the attitude and actions of artists (El-Or, p. 155). Other researchers take more flexible approaches. For archeologists such as Malinowski (2013, p. 80; Helm et al., 2001), style is the projection of cultural signals onto material artifacts and involves a choice among different alternatives (Hegmon, 1992, pp. 517-518).

Schapiro, for instance, defines style as the constant elements, qualities, and expression in the art of individuals or groups (1953). For Schapiro, style cannot be generalized, as it is a function of the observer. Schapiro's discussion reinforces our motivation to study style as a function of attributes (techniques and gestures) used over 
time, with respect to the maker and the image in action, seeking a common ground of similarities and differences as a platform for understanding makers' styles. For Schapiro, a historical-psychological view on style is still awaiting investigation.

Wiessner takes the first steps toward such a psychological view, defining style as formal variation in material culture that transmits information about identity (1983). Her theory connects variations in material culture to individuals' behavior and cognition, which is especially relevant to our investigation of the dynamic characteristics of style (1984. p. 191). We aim at introducing a rigorous, computational framework for investigating the dynamics of style as the first stage for a behavioral view of the making practice.

Style in the history of the arts. In the first half of the 20th century, the style was a dominant concern in art history (Nelson and Shiff, 2012, p. 98). The meanings and the means (techniques, materials, and esthetics) were separated, narrowing the definition of style to consistent esthetics (Helm et al., 2001). Later, Alpers explored art from a multidisciplinary perspective, trying to reveal the fundamental forces that made art what it is (1979).

Style and the digital world. Digital tracking techniques connect style to motor skills, from wearable sensors (Lapinski, 2013; Hoshen and Peleg, 2016) to smart tools (Zoran et al., 2014b). Such methods allow the investigation of style beyond its visual properties, through an examination of the dynamics that yielded them. A body of work has been dedicated to digitally extracting stylistic features from images (Sablatnig et al., 1998; Khan et al., 2010; Gatys et al., 2015). Elgammal et al. used convolutional artificial neural networks to classify large sets of paintings (2018), and Saleh et al. explored automated discovery of artistic influences (2016). In computer graphics, such tools are used for stylization-changing a graphical element in a way that preserves the content but brings its appearance closer to a reference (LunaPic, n.d.; Kyprianidis, 2013).

Recent works in the realm of digital craft combine real-world expertise with virtual spaces and call for a deeper investigation of the dynamics of style. Examples include Arisandi et al.'s framework for creating 3D models by hand using virtual reality (2012) and other works that take hybrid approaches to make objects (Zoran et al., 2014b; Devendorf et al., 2015; Shilkrot et al., 2015).

\section{Motor skills as dynamics of style}

Our computational model emphasizes the dynamic nature of the style, as time-dependent with multiple aspects. We define below the maker's dynamic style as the set of relevant properties and techniques, while the static style is a projection of the dynamic style at a specific time within the space of esthetics:

- The dynamic style denotes an understanding of style as comprised of a finite set of observable, measurable, analyzable, and interdependent motor acts within a realtime temporal order, and as employed in the process of a maker's creation. Such a definition of dynamic style allows for a quantitate analysis via computational tools of stylistic motor skills, as we propose in this paper.

- The static style is a singular interpretation of the maker's esthetics (most cases) analyzed from the finished work. It is a set of visual features rendered on a produced artifact.

We hypothesize that each maker uses a consistent set of motor skills. Such skills are embodied in the techniques the maker uses. With respect to such techniques, we observe a dynamic style in the making process via the statistical relationship between techniques and their transitions. Hence, we can observe the final outcome through the dynamics and causalities that contributed to it in the making process. For example, the tool's angle varies
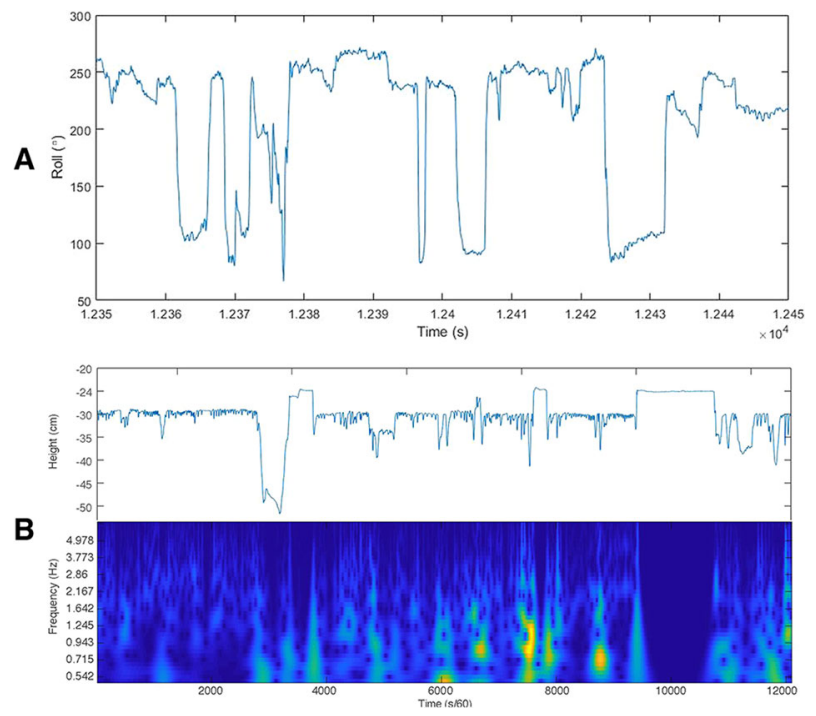

Fig. 1 Tool's roll angle and height features. A Example of the way a tool's roll angle changes while a maker works with it. B Part of the height signal and its corresponding CWT for frequencies greater than $0.5 \mathrm{~Hz}$.

between few dominant values (see Fig. 1A), making it possible to look at the sculptor's work as a (statistical) transition between a limited set of states (techniques).

We investigated combinations of dominant motor skills and how they vary among sessions, observing the making process as a transition in time between different motor skills. We tracked the usage of the sculptural tool, then analyzed the principal tool's positions and the transitions between them. The framework of all experiments consisted of the following elements:

i. We rely on clay relief technique, in which details are sculpted by manipulating a solid background of clay. This technique is particularly relevant for our work, being accessible to inexperienced participants, but requiring practice to master. Moreover, it allows both coarse and accurate modes of working.

ii. We use a knife-like sculpture tool with a motion-tracking sensor embedded in the handle. An experienced maker modeled its tip from epoxy (Fig. 2B). The tool can move freely in all directions.

iii. A Polhemus FASTRAK is used as the magnetic motiontracking device, allowing 6DOF tracked at $60 \mathrm{~Hz}$.

iv. Each session was executed in a $20 \times 20 \times 4 \mathrm{~cm}$ box-like terracotta clay body. Each was produced using the same square mold and left in a loose nylon bag to solidify for $24 \mathrm{~h}$ (Fig. 2C, D).

v. For all experiments, we used a similar light setting in the lab (Fig. 2A).

The research and the following studies have been approved by the Ethics Committee for Non-Medical Human Studies in the Faculty of Natural Sciences of the Hebrew University of Jerusalem (26052021). We confirm that informed consent was obtained from all participants and/or their legal guardians. The datasets generated and analyzed during the current study are not publicly available to protect participants privacy but are available from the corresponding author on reasonable request.

\section{Algorithmic framework}

To identify the main tool's states, we characterized each time sample with specific features and extracted the states from the 

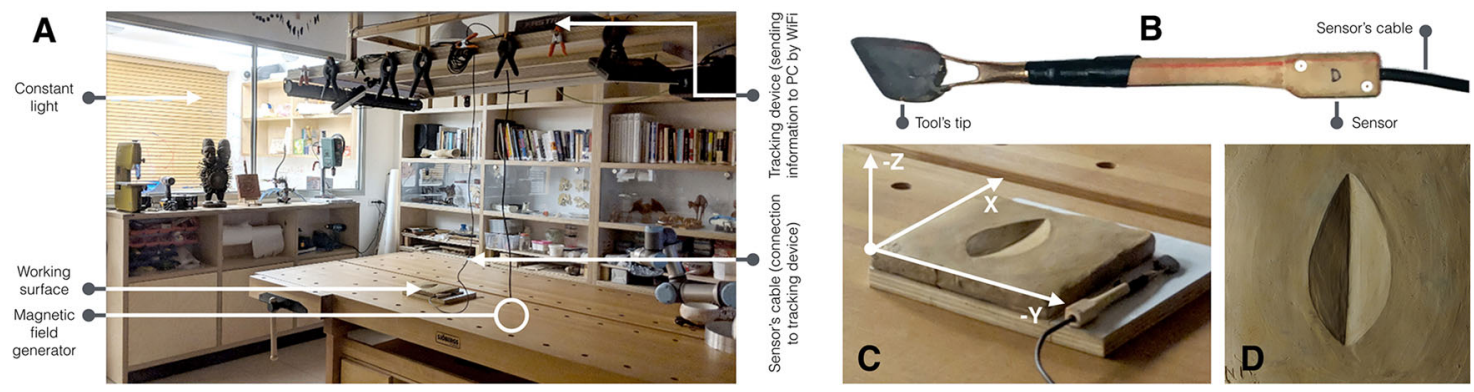

Fig. 2 The lab's settings during the experiments. A The participants' workspace, including the tracking device, and the constant light configuration from the side room. B Main elements of the tool, including the tip, which does all of the clay manipulations; the sensor location; and the power cable for the sensor. C-D An example of the clay body: a terracotta variety of grayscales formed by light and orientation.

normalized features space. Our data analysis consists of continuous wavelets transform (CWT), and unsupervised machine learning (k-Means and Gaussian mixture model, GMM). The use of such concepts for behavior analysis can be found in other works (Linden et al., 2003; Lapinski, 2013).

Features space. To define which features should be quantitatively measured, we characterize the tool's dynamics, focusing on the maker's typical motor skills. We characterized the tool's state by its height, orientation, and signature of its local behavior at each time sample. The transitions between different parts on the working surface can also reveal significant information.

i. Height: The height of the tool is approximately the tool-tip's $\mathrm{Z}$ coordinate (as the sensor is on the other side of the tool). This feature contains data such as the depth of the relief or distribution between different heights throughout the process, including during idle working modes. Figure 1B shows part of the tool's height signal and the corresponding CWT in the range of relevant frequencies. In the data analysis of our presented work, we used only the high frequency of the height feature.

ii. Euler angles: These features are connected to a variety of aspects, including the angle of the plane currently being manipulated and the makers' preference for which part of the tool's tip to work with. Due to the cyclic nature of angles, an artificial discontinuity point occurs when crossing the feature's range of the original signal. We handle this phenomenon in two different ways: the first is embedding the signal in a $2 \mathrm{D}$ unit circle, and the second is minimizing the number of these discontinuity points by cyclically shifting the signal in its original range. The method used depends on the clustering method in further stages in the algorithm: we use the embedding method when using $\mathrm{k}$ means, and shift the signal when using GMM.

iii. High-frequency features: As an indication of the local behavior of a feature, we calculated its high-frequency corresponding feature. The high-frequency feature for a feature $f$ is the absolute of the continuous 1-D wavelet transform coefficients of $f$, corresponding to frequencies greater than a threshold $s=0.5 \mathrm{~Hz}$, projected into smaller dimensionality space using PCA, such that $85 \%$ of the variance is explained.

Normalization of the feature space: Different features needed to be normalized before clustering the data. Given a feature, we transform each sample to distribution between its main clusters, making our feature space a space of distributions between different clusters of each feature $f$. First, we estimated the number of clusters in $f$. As the number of clusters is not a well-defined property of the data, by using both Elbow methods and Silhouette criteria variants, we decided that three clusters for regular features and five for high-frequency features will be a reasonable choice. We then clustered the data and transformed each sample to distribution between the clusters of the signal.

Overall, we used two main strategies for establishing the features space:

i. GMM-based analysis. We fixed the angular features by shifting, then used GMM on each feature in order to transform each sample to a distribution.

ii. $k$-Means based analysis. We embedded the angular features in the 2D unit circle, then used k-Means to transform each sample to distribution between the main clusters.

As we were interested in both methods, we checked both for the establishment of the feature space. When a GMM variant was used for clustering, the posteriors are part of the standard output. When k-Means was used, the probability for the sample $f_{i}$ to belong to cluster $j$ with center $c_{j}$ is proportional to $d_{i, j}^{-1}$, where $d_{i, j}=\left|f_{i}-c_{j}\right|$.

The principal tool's states: For each experiment, we used $\mathrm{k}$ Means to cluster the normalized feature space to $\hat{k}$ clusters, explaining $90 \%$ of the variance (see. Fig. 3A).

Comparing between different working sessions. After the previous step, each time slice can be regarded as a series of principal working states (see Fig. 4). For exploring motor skills in creative processes from a statistical point of view, we transformed a working series in time to distribution between the $\hat{k}$ working states of feature space, and the probabilities of changing from one working set to another between them. We used the JensenShannon divergence to quantify the difference between time segments according to the distribution between the working states, the distribution of transitions between the working states, and both distributions.

\section{Short-term tasks by unskilled participants}

We assume that even unskilled makers have a consistent set of working states, and examine whether these can be detected using our proposed framework. To validate this assumption and examine the robustness of our method, we conducted an experiment involving a group of five unskilled participants; all had some experience with craft techniques, but none had worked with clay relief.

All participants performed three relatively simple tasks with increasing complexity, executing a specific geometric pattern for each: a two-sided, leaf-shaped ornament; a circular ornament divided into four equal sections; and a purely circular ornament (see Fig. 5A-C).

Each session lasted $30 \mathrm{~min}$, and we notified the participants of the time remaining at 15, 25, and $29 \mathrm{~min}$. Each participant waited for one to two weeks between tasks. At each session, a physical one-to-one model of the desired output was located next to the participant, and a sketch of the desired pattern appeared on the clay surface (see Fig. 5D). 


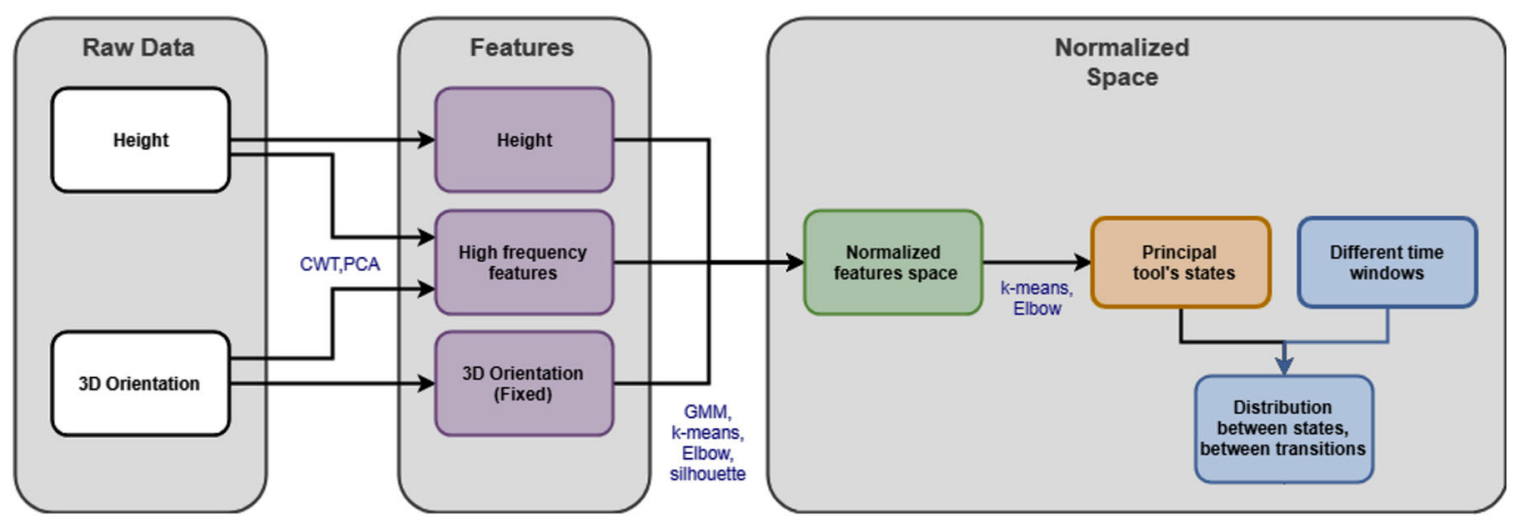

Fig. 3 The algorithmic workflow of our data analysis. The main algorithms used in each step are in blue.
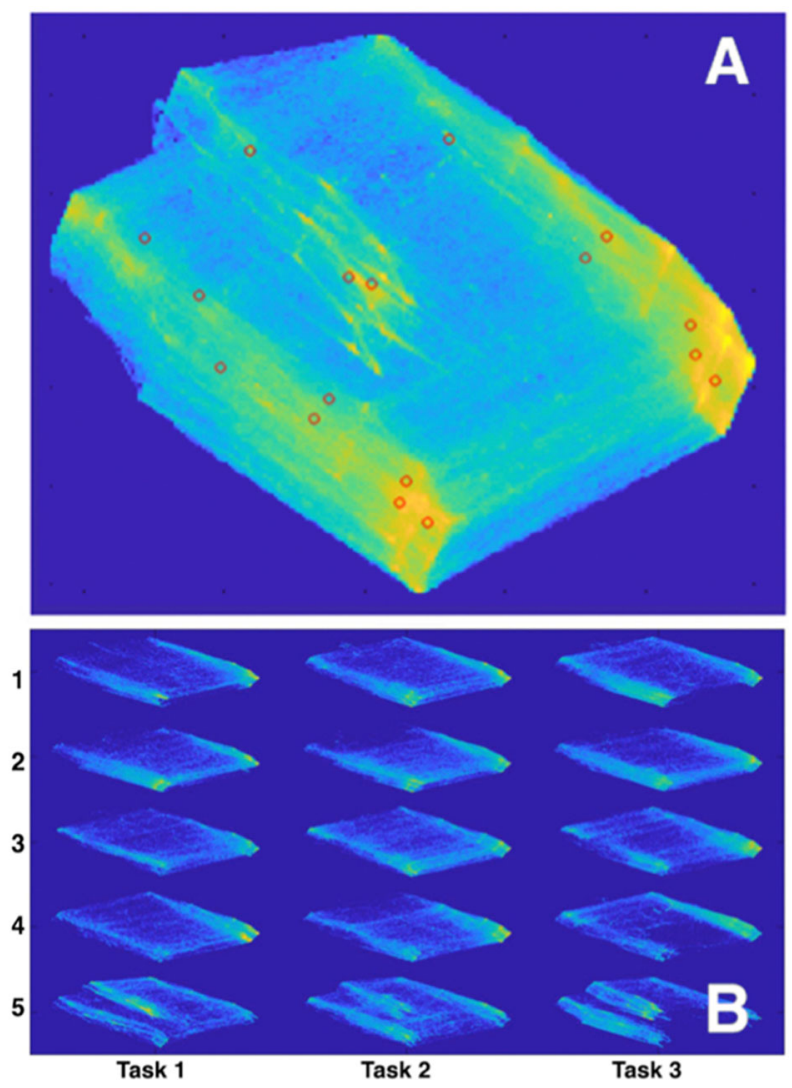

Fig. 4 Features space histograms. A $200 \times 200$ histogram (log-scaled) of the normalized features space projected into 2D using PCA, and principal tool's states in the normalized features space. B Each session's contribution to the $2 \mathrm{D}$ histogram of the entire normalized features space.

We calculated the distances (as explained in the "Algorithmic framework" section) between the working sessions, repeating this process 10 times; averaged the distance matrices; and embedded the sessions in $2 \mathrm{D}$ using the averaged distance matrix and nonclassical MDS (see Fig. 7A).

Fig. 4B shows a 2D histogram of each session's contribution to the 2D histogram of the entire normalized features space. Participant 5 demonstrates an individual set of different tool states, while the four remaining participants are relatively similar, although participant 1 visits 5's unique set of tool states from time to time, and 4's contribution exists mainly in the upper right of the histogram.

The results emphasize not only that the unskilled participants are relatively consistent in their motor skills and the use of the
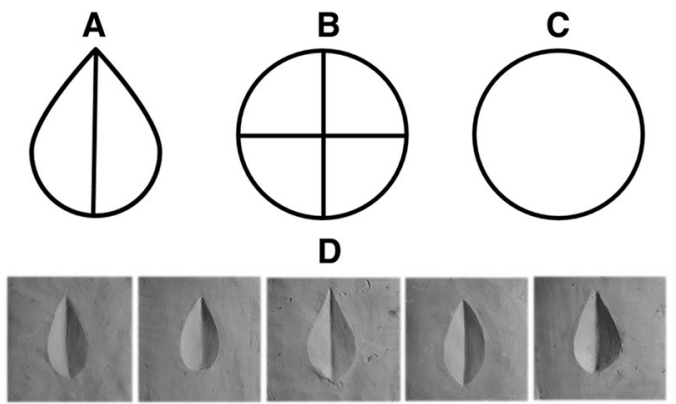

Fig. 5 The first experiment. A-C Diagrams of the first experiment's sculpting tasks. D Reliefs from the first experiment's first task.

tool, but that these similarities can be detected by the morphology (Fig. 4B) of the field participants' span by their motoric actions, and the distance between such fields (Fig. 7A). The formal representation of dynamic style as a function of features extracted from ones' motor actions generates both inner field analytics (the morphology of one's field of motoric actions) and external, relative analytics (compression between others), an observation that can be used for temporal and relative classifications of one's working style, per subject matter.

\section{Long-term process of a skilled sculptor}

Within this experiment, we investigate whether we can differentiate between sets of techniques defined by their set of features when observing an experienced maker. This involves an in-depth study with a single skilled participant, a professional sculptor who teaches clay relief at a leading art academy.

The participant performed four advanced, long-term tasks: two translation tasks of a 3D object (flower) to relief, and two flower-like geometric patterns (see Fig. 6). Both required the participant to produce a number of areas with different orientations, and interpret a $3 \mathrm{D} / 2 \mathrm{D}$ image to $2.5 \mathrm{D}$. Yet some challenges are different: in the flowers tasks ( $3 \mathrm{D}$ to $2.5 \mathrm{D}$ ), the image is less symmetric and more organic, and the task calls for additional decisions regarding organization and interpretation. In the patterns tasks (2D to $2.5 \mathrm{D})$, the image and process are more structured.

The whole process was divided into seven weekly meetings of two hours each, with a 30-min session dedicated to each task, allowing a total of $3.5 \mathrm{~h}$ for each one. In each meeting, the order of the tasks was different. We calculated the distances between all the working sessions, repeated this process for 10 repetitions, averaged the distance matrices, and embedded the sessions in $2 \mathrm{D}$ using the averaged distance matrix and non-classical MDS (see Fig. 7B). 


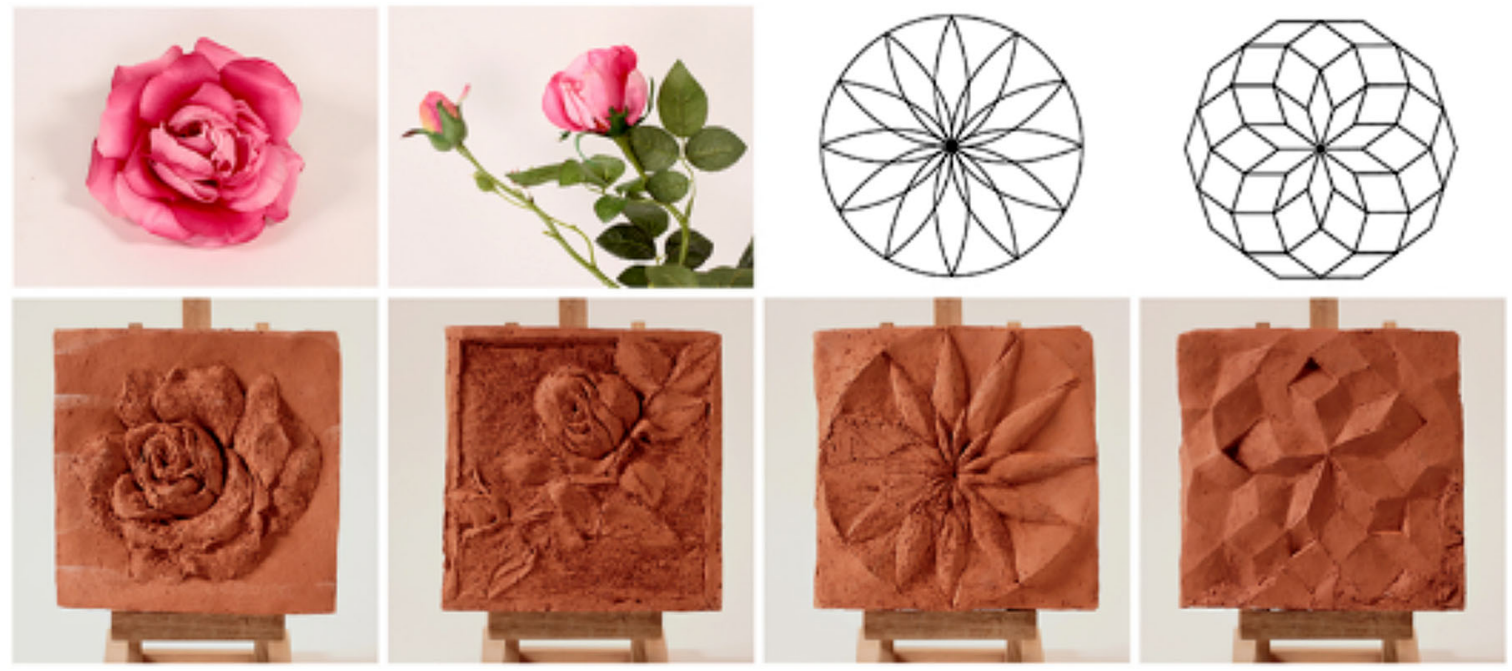

Fig. 6 The second experiment. The experiment's sculptural tasks (above) and final reliefs (below).

2D embedding of all novice sessions, GMM analysis

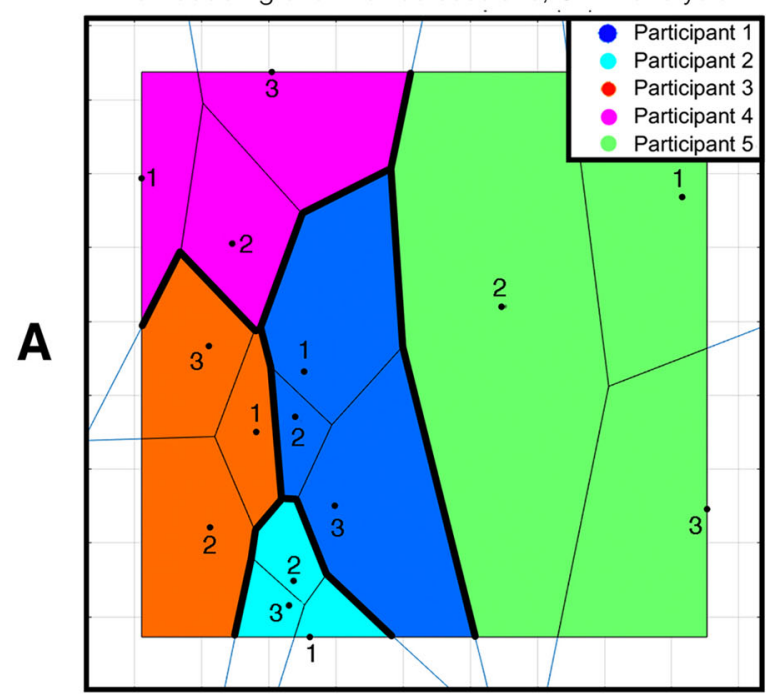

2D embedding of all expert sessions, GMM analysis

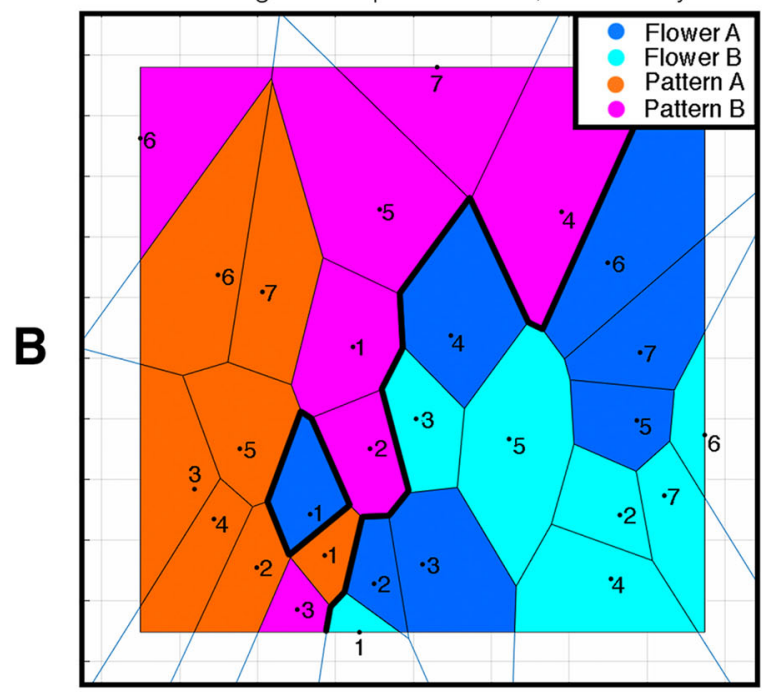

2D embedding of all novice sessions, k-Means analysis

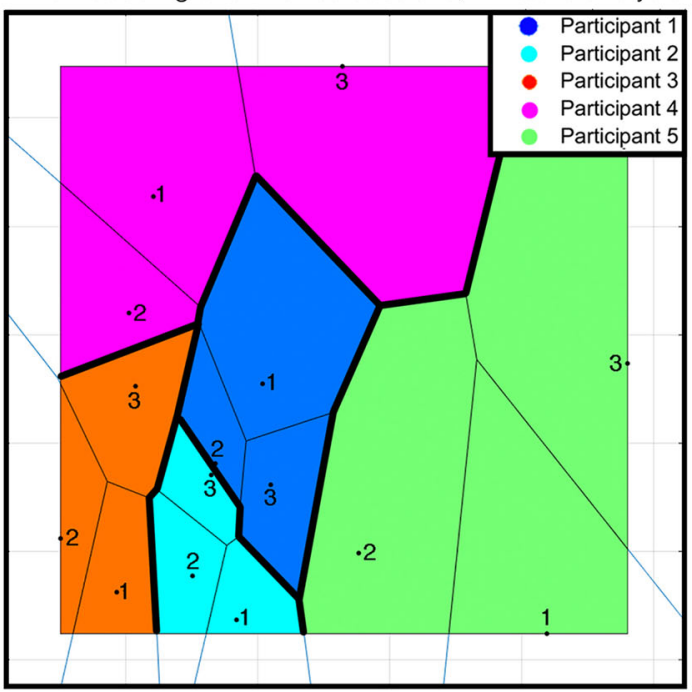

2D embedding of all expert sessions, k-Means analysis

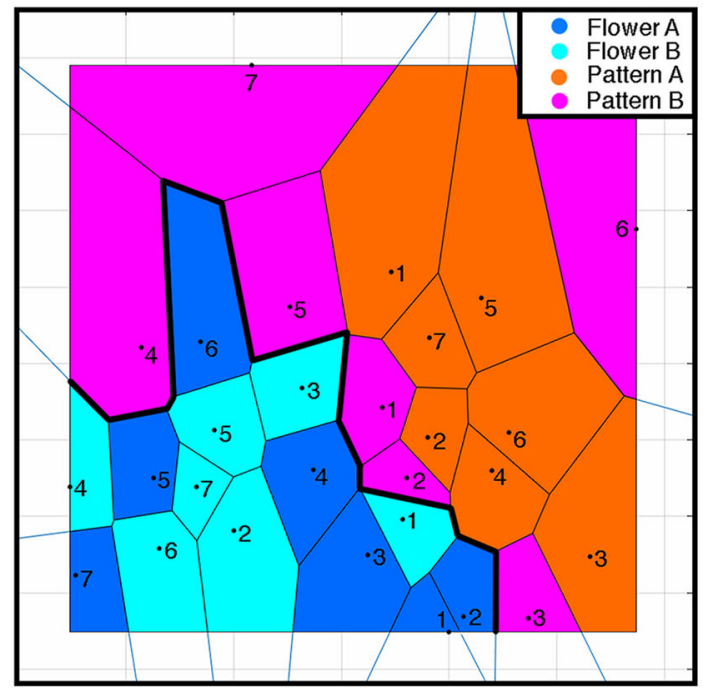

Fig. 7 2D embedding of all experiments. Embedding of all (A) novice and (B) expert sessions, distance based on the distributions of both states and transitions, and the Voronoi diagram of the embedding based on both GMM and k-Means analysis. 
When the sessions are embedded in $2 \mathrm{D}$, there is a clear separation between the two groups (flowers vs. patterns). This suggests that the features collected from the work, and the corresponding computational analysis give sufficient indications for differentiation in dynamic style. Such various sections of dynamic style are correlated with the categories of the work (flowers vs. patterns).

Nevertheless, the two groups do not form two separate clusters inside each group. The distinctions in the flowers' images with respect to the patterns (Fig. 6) contributes to a less consistent dynamic style in the flower, as demonstrated in Fig. 7B, as the last presents a mapping of relative distances between the set of techniques used in each task-the closer the tasks in the figure, the more they represent a similar dynamic style.

It is important to state the autonomous, unbiased means of such detection; thus, correlations are found solely based on the set of a participant's motoric actions and the way these actions correspond to each other. A direct deduction of this finding is that a group of motor actions (techniques) as represented by a set of technical features can be directly linked to higher-level representation and classification the artist applies on the models of her work. In other words, the tracking and analyzing of one's motoric actions using high temporal resolution hold cognitive information about higherlevel interactions between the artist and the model.

\section{Discussion}

With respect to our earlier definition of dynamic style, we selected a finite set of observable and measurable features derived from the motoric actions of each participant, such as a tool's temporal angle and its position frequency. The analysis of the time-variant behaviors of these features results in a state of classifications or working techniques. From our first experiment, we see that automatic, untagged analysis of the techniques and the temporal interplay between techniques shows that a typical, personal dynamic style can be recognized. Hence, such temporal features can be studied as basic qualities for the classification of one's dynamic style, and thus can inform one's work dynamics using correlations between various working sessions, between sessions from the same work, or between different artists.

In addition, our second experiment shows that a similar analysis of a long-term process of a skilled sculptor reveals correlations between the sculptor model of the work and her (unaware) preference for a set of motoric actions used for the task. From a theoretical perspective, the techniques embedded in the features space, and the temporal interplay between techniques, reveal information about the artist's subjective correspondence to and association with the model of the work.

\section{Conclusions}

The two experiments reinforce our approach of observing style as a transition between working states, provide a summary of the making process, and open the door to computational frameworks in style research. We perceived different aspects of making: the consistency and individuality of makers, and how different models and sub-techniques affect the making process. While additional work is required to generalize conclusions regarding the nature of the personal style, our computational framework has been shown to be rigorous and robust, proving the concept of our computational approach.

The main contribution of this work is the analysis and definition of dynamic style as the time-dependent motoric functions that ultimately, upon completion of the artist's making process, produce the static style in a finished work. This allows for the making process as a whole to be quantitatively observed as an interplay between various actions regardless of the esthetic qualities of the artifact.
Such an approach to dynamic style raises themes for investigation:

- The connection between time and style. Different aspects of makers' styles arise over different timescales. Different elements from different timescales are connected, and a future model of dynamic style should allow for the analysis of the characteristics of the progression of style as a function of time.

- The connection between style, technique, and skill. Our work suggests that the correlation between technique and motor skills is not given but dynamic; it evolves over time and probably reflects a complex dependency in both the mental and motor state of the maker.

- The maker's perception, image, and esthetics. As the maker's mind and set of mental images evolve over time, it is worth exploring the connections between the maker's mental states and the sets of images and esthetics used in their work.

- Relationship between cultural and personal esthetics. Cultural style may restrain and bias the esthetics of the individual, whose personal style, in turn, enriches their cultural style. Yet the relationship between the two is complex and should be explored further.

As the topics above are open theoretical questions not easy to study quantitatively, we suggest focusing on the following issues, which are well suited for computational analysis:

i. How does one's technique and skill change over short-term and long-term artistic processes? In what ways are makers (not) consistent between different artistic techniques?

ii. What technical features are essential when exploring the maker's personal style, and to what extent are motor actions essential for characterizing the maker's esthetics?

iii. Does iconography affect a maker's motor actions or technique during the making process?

In addition to these investigations, there are broader theoretical and ethical concerns regarding modeling and (computationally) imitating one's style. As mentioned briefly earlier, in the age of ML and deep learning, it is reasonable to assume that capturing a novice's style will allow a prediction of their artistic skills, assuming (hypothetically) that they will continue to practice. Having such a capability may raise a new discourse regarding an entirely new form of HRI: interacting with a skillful version of yourself. Can a user guide a robotic agent working like she or he may work in the future, without having acquired the technical skill yet? Does human agency and ownership of creative practice depend on the process of acquiring these creative skills? While such questions may never be answered, they can shape the way we envision a future HRI for creative tasks, hybrid applications, and human-machine co-creation.

Received: 19 February 2020; Accepted: 1 June 2021; Published online: 29 June 2021

\section{References}

Alpers S (1979) Style is what you make it: the visual arts once again. In:Lang B (ed) The Concept of Style. University of Pennsylvania Press, Philadelphia, pp. 137-62

Alves-Oliveira P, Paiva A, Arriaga P, Hoffman G (2017) Yolo, a robot for creativity: a co-design study with children. IDC 2017. In: Proceedings of the 2017 ACM Conference on Interaction Design and Children, Stanford, California, pp. 423-429

Arisandi R, Takami Y, Otsuki M, Kimura A, Shibata F, Tamura H (2012). Enjoying virtual handcrafting with ToolDevic. In: UIST Adjunct Proceedings '12 Adjunct Proceedings of the 25th Annual ACM Symposium on User Interface Software and Technology, Cambridge, Massachusetts, pp. 17-18 
Barthes R (1977) The death of the author. In: Barthes R, Image, Music, Text (trans: Heath S), Hill and Wang, New York, pp. 142-148

Benjamin W (2018) The work of art in the age of mechanical reproduction. In: Barnes AJ, Bunning K, Watson A (eds) A Museum Studies Approach to Heritage. Routledge, Abingdon, pp. 226-243

Boas F (1995) Primitive art. Dover Publications, New York

Clark K (2015) Civilisation. John Murray, London

Cobbledick S (1996) The information-seeking behavior of artists: exploratory interviews. Library Quart 66:343-372

Davis W (1990) Style and history in art history. In: Conkey MW, Hasdorf CA (eds) The Uses of Style in Archaeology, Cambridge University Press, pp. 18-31

de Miranda L, Ramamoorthy S, Rovatsos M (2016) We, Anthrobot: Learning from Human Forms of Interaction and Esprit de Corps to Develop More Plural Social Robotics. Front Artificial Intell Applicat 290:48-59. https://doi.org/ 10.3233/978-1-61499-708-5-48

Devendorf L, Ryokai K (2015) Being the machine: reconfiguring agency and control in hybrid fabrication. In: CHI '15 Proceedings of the 33rd Annual ACM Conference on Human Factors in Computing Systems, Seoul, Republic of Korea, April 2015, pp. 2477-2486

Elgammal A, Mazzone M, Liu B, Kim D, Elhoseiny M (2018) The shape of art history in the eyes of the machine. Proc AAAI Conference Artificial Intelligence https://arxiv.org/abs/1801.07729v2

El-Or T (2014) Sandals: the anthropology of local style. Am Oved, Tel Aviv

Gandon E, Bootsma RJ, Endler JA, Grosman L (2013) How can ten fingers shape a pot? Evidence for equivalent function in culturally distinct motor skills. PLoS ONE 8:e81614. https://doi.org/10.1371/journal.pone.0081614

Gatys LA, Ecker AS, Bethge M (2015) A neural algorithm of artistic style. arXiv. https://arxiv.org/abs/1508.06576

Ghahramani Z (2015) Probabilistic machine learning and artificial intelligence. Nature 521(7553):452-459. https://doi.org/10.1038/nature14541

Glazek K (2012) Visual and motor processing in visual artists: Implications for cognitive and neural mechanisms. Psychol Aesthet Creat Arts 6:155-167

Gombrich EH (1995) The story of art, 16th ed. Phaidon Press, London

Görür OC, Rosman BS, Hoffman G, Albayrak S (2017) Toward integrating Theory of Mind into adaptive decision-making of social robots to understand human intention. Paper presented at the 12th ACM/IEEE International Conference on Human-Robot Interaction (HRI), Vienna, Austria, 6 March 2017

Haroch O, Glauber N, Zoran A, Grosman L (2019) On quantifying and visualizing the Potter's personal style. J Archeol Sci 108:104973. https://doi.org/10.1016/j. jas.2019.104973

Hegmon M (1992) Archaeological research on style. Ann Rev Anthropol 21:517-536

Helm AA, Boas F, Malinowsky B (2001) A contrast, comparison, and analysis. Lambda Alpha J Man 31:42-51

Hoffman G, Weinberg G (2010) Gesture-based human-robot jazz improvisation. In: Proceedings of the IEEE International Conference on Robotics and Automation, Anchorage, Alaska, pp. 582-587

Hoshen Y, Peleg S (2016) An egocentric look at video photographer identity. In: 2016 IEEE Conference on Computer Vision and Pattern Recognition (CVPR), Las Vegas, Nevada, pp. 4284-4292

Jay F, Renou J-P, Voinnet O, Navarro L (2017) Unpaired Image-to-Image Translation using Cycle-Consistent Adversarial Networks Jun-Yan. In: Proceedings of the IEEE International Conference on Computer Vision, Venice, Italy, pp. 183-202

Khan FS, van de Weijer J, Vanrell M (2010) Who painted this painting? In: The 2010 CREATE Conference, Williamsburg, Virginia, pp. 329-33

Kozbelt A (2001) Artists as experts in visual cognition. Visual Cogn 8:705-723. https://doi.org/10.1080/13506280042000090

Kyprianidis JE, Collomosse J, Wang T, Isenberg T (2013) State of the "art": a taxonomy of artistic stylization techniques for images and video. IEEE Trans Visualization Comput Graphics 19:866-885

Lapinski MT (2013) A platform for high-speed biomechanical analysis using wearable wireless sensors. Dissertation, Massachusetts Institute of Technology

Linden G, Smith B, York J (2003) Amazon.com recommendations: item-to-item collaborative filtering. IEEE Internet Comput 7(1):76-80. https://doi.org/ 10.1109/MIC.2003.1167344

LunaPic.com (2020) Photo Editor Van Gogh tool. Lunapic; https://www.lunapic. com/editor/?action=vangogh, Accessed 15 Aug 2020

Mace M, Ward TB (2002) Modeling the creative process: a grounded theory analysis of creativity in the domain of art making. Creativity Res J 14 (2):179-192. https://doi.org/10.1207/S15326934CRJ1402_5

Malinowski B (2013) Argonauts of the Western Pacific: An Account of Native Enterprise and Adventure in the Archipelagoes of Melanesian New Guinea. Enhanced Edition. Waveland Press, Long Grove, Illinois

Mordvintsev A, Olah C, Deepdream MT (2015) https://github.com/google/ deepdream. Accessed 15 August 2020

Nelson RS, Shiff R (2012) Critical terms for art history. University of Chicago Press

Nikolaidis S, Shah J (2012) Human-Robot Teaming using Shared Mental Models. Paper presented at ACM/IEEE International Conference on Human Robot Interaction (HRI), Workshop on Human-Agent-Robot Teamwork, Boston, Massachusetts
Rigau J, Feixas M, Sbert M (2008) Informational dialogue with van Gogh's paintings. In: Brown et al. (eds) Proceedings of the Fourth Eurographics Conference on Computational Aesthetics in Graphics, Visualization and Imaging (Computational Aesthetics'08), Lisbon, Portugal, pp. 115-122

Sablatnig R, Kammerer P, Zolda E (1998) Hierarchical classification of paintings using face- and brush stroke models. In: ICPR'98 Proceedings of the 14th International Conference on Pattern Recognition-Volume 1 (IEEE Computer Society), Plymouth, UK, 23-25 November 1998, pp. 172

Saleh B, Abe K, Arora RS, Elgammal A (2016) Toward automated discovery of artistic influence. Multimedia Tools Applicat 75:3565-3591

Schapiro M (1953) Style, in Kroeber AL (ed) Anthropology today. An Encyclopedic Inventory, University of Chicago Press, pp. 287-312

Shilkrot R, Maes P, Paradiso JA, Zoran A (2015) Augmented airbrush for computer aided painting (CAP). ACM Trans Graphics 34:1-11

Solso RL (2001) Brain activities in a skilled versus a novice artist: an fMRI study. Leonardo 34(1):31-34

Stevens V (2014) To think without thinking: the implications of combinatory play and the creative process of neuroaesthetics. Am J Play 7(1):99-119

Stevenson A (ed) (2010) Oxford Dictionary of English. Oxford University Press

Summers D (2009) Style. In: Preziosi D (ed) The art of art history: a critical anthology. New Edition. Oxford University Press, pp. 144-148

Thomaz A, Hoffman G, Cakmak M (2016) Computational human-robot interaction. Foundations Trends Robotics 4(2-3):104-223. https://doi.org/10.1561/ 2300000049

Wiessner P (1983) Style and social information in Kalahari San projectile points. Am Antiquity 48:253-276

Wiessner P (1984) Reconsidering the behavioral basis for style: a case study among the Kalahari San. J Anthropol Archaeol 3:190-234

Winner E, Casey MB (1992) Cognitive profiles of artists. In: Cupchik GC, László J (eds), Emerging Visions of the Aesthetic Process: In Psychology, Semiology, and Philosophy, Cambridge University Press, pp. 154-170

Zoran A (2016) A manifest for digital imperfection. XRDS 22(3):22-27. https://doi. org $/ 10.1145 / 2893491$

Zoran A, Shilkrot R, Goyal P, Maes P, Paradiso JA (2014a) The wise chisel: the rise of the smart handheld tool. IEEE Pervasive Comput 13(3):48-57

Zoran A, Shilkrot R, Nanyakkara S, Paradiso J (2014b) The hybrid artisans: a case study in smart tools. ACM Trans. Comput.-Hum. Interact 3:15. https://doi. org/10.1145/2617570. 1-15:29

\section{Acknowledgements}

We would like to thank all the participants in our experiments, with special regards to D.K. Gueller; all the participants in the study; members of the Hybrid Lab; Prof. R. Fattal; Prof. A. Wiesel; Prof. L. Grosman; and Dr. M. Gavish for their advice and support. Special thanks to Dr. K. Goltseker for the helpful scientific comments during different stages of this work This work could not have been finalized without the help of R. Rosner Kontorovich and C. Evers, and we are grateful to them for putting so much effort into editing this document. The work was partially supported by the Israeli Science Foundation (ISF) in its early stage and mainly supported by The HUJI Federmann Cyber Security Center.

\section{Competing interests}

The authors declare no competing interests.

\section{Additional information}

Correspondence and requests for materials should be addressed to A.R.Z.

Reprints and permission information is available at http://www.nature.com/reprints

Publisher's note Springer Nature remains neutral with regard to jurisdictional claims in published maps and institutional affiliations.

Open Access This article is licensed under a Creative Commons Attribution 4.0 International License, which permits use, sharing, adaptation, distribution and reproduction in any medium or format, as long as you give appropriate credit to the original author(s) and the source, provide a link to the Creative Commons license, and indicate if changes were made. The images or other third party material in this article are included in the article's Creative Commons license, unless indicated otherwise in a credit line to the material. If material is not included in the article's Creative Commons license and your intended use is not permitted by statutory regulation or exceeds the permitted use, you will need to obtain permission directly from the copyright holder. To view a copy of this license, visit http://creativecommons.org/ licenses/by/4.0/

(c) The Author(s) 2021 